KODAI MATH. SEM. REP.

21 (1969), 151-159

\title{
ON INFINITESIMAL DEFORMATIONS OF CLOSED HYPERSURFACES
}

\author{
By Yosio Mutō
}

\section{§ 1. Introduction.}

In the present paper we study the effect of infinitesimal deformations of (1) a closed orientable hypersurface in an orientable Riemannian manifold and (2) a closed hypersurface in a Euclidean space on some integrals.

Let $M$ be an $(n+1)$-dimensional orientable Riemannian manifold and $M^{\prime}$ be a closed orientable hypersurface in $M$ whose equations are given by

$$
x^{h}=x^{h}\left(u^{a}\right)
$$

in local coordinates. We use indices $h, i, j, k$ for $M$ and $a, b, c, d$ for $M^{\prime}$, hence $h, i, j, k$ run over the range $\{1, \cdots, n+1\}$ and $a, b, c, d$ over the range $\{\dot{1}, \cdots, \dot{n}\}$. As usual $B_{a}{ }^{h}$ means $\partial_{a} x^{h}$ where $\partial_{a}=\partial / \partial u^{a} . \quad g_{b a}=B_{b}{ }^{i} B_{a}{ }^{h} g_{i h}=B_{b a}^{i h} g_{i h}$ are the components of the first fundamental tensor of $M^{\prime}$. The unit normal vector is denoted by $N^{h}$ and the reciprocal of the matrix $\left(B_{a}{ }^{h}, N^{h}\right)$ by $\left(B^{a}{ }_{h}, N_{h}\right) . \quad \nabla$ means the Van der Waerden-Bortolotti differential operator, hence $\nabla_{b} B_{a}{ }^{h}=h_{b a} N^{h}, \nabla_{b} N^{h}=-h_{b}{ }^{a} B_{a}{ }^{h}$ where $h_{b}{ }^{a}=h_{b c} g^{c a}$. $h_{b a}$ are the components of the second fundamental tensor of $M^{\prime}$.

\section{$\S 2$. Infinitesimal deformations.}

Let $\mathscr{M}^{\prime}$ be a set of hypersurfaces $M^{\prime}(t), 0 \leqq t<\varepsilon$, where $\varepsilon$ is a sufficiently small positive number and $M^{\prime}(0)=M^{\prime}$. We assume that the local coordinates of the points of $M^{\prime}(t)$ are given by

$$
x^{h}=x^{h}\left(u^{a}, t\right)
$$

in $M$. We also assume that $x^{h}\left(u^{a}, t\right)$ are $C^{\infty}$ functions and the mapping $\varphi(t)$ : $M^{\prime}(0) \rightarrow M^{\prime}(t)$ induced by

$$
x^{h}\left(u^{a}, 0\right) \rightarrow x^{h}\left(u^{a}, t\right)
$$

is diffeomorphic, $u^{a}$ being local coordinates of $M^{\prime}(t)$ in $U \cap M^{\prime}(t)$ for some neighborhood $U$ of $M$ and for all $t \in[0, \varepsilon), \varphi(t)$ is a deformation of $M^{\prime}$.

We define $\xi^{h}\left(u^{a}\right)$ by

Received September 5, 1968. 


$$
\xi^{h}\left(u^{a}\right)=\left(\partial_{t} x^{h}\left(u^{a}, t\right)\right)_{0}
$$

where $\partial_{t}=\partial / \partial t$ and ()$_{0}$ means ()$_{t=0} . \xi^{h}\left(u^{a}\right)$ is the vector field of an infinitesimal deformation.

The infinitesimal deformation $D g_{b a}$ of the metric of $M^{\prime}$ associated with the vector field $\xi^{h}$ will be denoted by $D_{b a}$. If $g_{b a}(u, t)$ is the metric tensor of $M^{\prime}(t)$, $D_{b a}$ is given by

$$
D_{b a}=\left(\partial_{t} g_{b a}(u, t)\right)_{0}
$$

for we have (2.1). Since $g_{b a}(u, t)$ is given by

$$
g_{b a}(u, t)=\partial_{b} x^{2}(u, t) \partial_{a} x^{h}(u, t) g_{i h}(x(u, t))
$$

we obtain

$$
D_{b a}=\partial_{b} \xi^{i} B_{a}{ }^{h} g_{i h}+B_{b}{ }^{i} \partial_{a} \xi^{h} g_{i h}+B_{b a}^{i h} \xi^{j} \partial_{j} g_{i h}
$$

hence

$$
D_{b a}=\nabla_{b} \xi^{i} B_{a}{ }^{h} g_{i n}+\nabla_{a} \xi^{i} B_{b}{ }^{h} g_{i h} .
$$

If $N^{h}(u, t)$ is the unit normal vector field of $M^{\prime}(t)$, we have

$$
\partial_{a} x^{i}(u, t) g_{i h}(x(u, t)) N^{h}(u, t)=0,
$$

hence

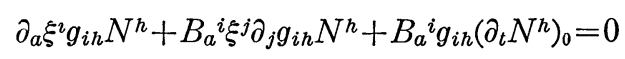

on $M^{\prime}$. From this we obtain

$$
N_{i} \nabla_{a} \xi^{2}+B_{a}^{i} g_{i n} P^{h}=0
$$

where $P^{h}$ is defined by

$$
P^{h}=\left(\partial_{t} N^{h}\right)_{0}+\left\{\begin{array}{c}
h \\
k j
\end{array}\right\} \xi^{k} N^{\jmath}
$$

on $M^{\prime}$ and will be called the infinitesimal deformation of the unit normal vector. From (2.5) and $N_{i} P^{i}=0$ we obtain

$$
P^{h}=-B_{a}{ }^{h} N^{i} \nabla^{a} \xi_{i}
$$

§3. Deformation of some integrals on a hypersurface in a Riemannian manifold.

Let us first consider the total volume of $M^{\prime}$,

$$
V=\int_{M^{\prime}} d V
$$


where $d V=\left(\operatorname{det}\left(g_{b a}\right)\right)^{1 / 2} d u^{1} \cdots d u^{n}$. It is known that the infinitesimal deformation of this integral, namely,

$$
D \int_{M^{\prime}} d V=\left[\frac{d}{d t} \int_{M^{\prime}(t)} d V\right]_{0}
$$

is given by

$$
\frac{1}{2} \int_{M^{\prime}} g^{b a} D_{b a} d V
$$

Substituting (2.4) into (3.1) and using Green's theorem, we get

$$
\begin{aligned}
\int_{M^{\prime}} B_{a}^{i} \nabla^{a} \xi_{i} d V & =-\int_{M^{\prime}} \xi_{i} \nabla^{a} B_{a^{2}} d V \\
& =-\int_{M^{\prime}} h_{a}{ }^{a} \xi_{i} N^{i} d V,
\end{aligned}
$$

hence

$$
D \int_{M^{\prime}} d V=-\int_{M^{\prime}} h_{a}^{a} N_{i} \xi^{2} d V
$$

Thus we obtain the following proposition.

Proposition 3.1. Let $M^{\prime}$ be a closed orientable hypersurface in an orientable Riemannian manifold. A necessary and sufficient condition that the total volume of $M^{\prime}$ be critical for every infinitesimal deformation such that

$$
\int_{M^{\prime}} N_{i} \xi^{2} d V=0
$$

is that the mean curvature be constant on $M^{\prime}$.

Let us calculate the deformation $D h_{b a}$ of the second fundamental tensor.

Let $h_{b a}(u, t)$ be the second fundamental tensor of $M^{\prime}(t)$. Since we have

$$
h_{b a}(u, t)=-B_{b}{ }^{2} g_{i h} \nabla_{a} N^{h}
$$

in $M^{\prime}(t)$, we get

$$
\partial_{t} h_{b a}(u, t)=-\left(\nabla_{b} \partial_{t} x^{i}\right) g_{i h} \nabla_{a} N^{h}-B_{b}{ }^{\imath} g_{i h}\left(\partial_{t} \nabla_{a} N^{h}+\left\{\begin{array}{c}
h \\
k j
\end{array}\right\} \partial_{t} x^{k} \nabla_{a} N^{\jmath}\right)
$$

By straightforward calculation we get 


$$
\begin{aligned}
\partial_{t} \nabla_{a} N^{h} & =\partial_{t}\left(\partial_{a} N^{h}+\left\{\begin{array}{c}
h \\
j i
\end{array}\right\} B_{a}^{j} N^{i}\right) \\
& =\partial_{a} \partial_{t} N^{h}+\partial_{t} x^{k} \partial_{k}\left\{\begin{array}{c}
h \\
j i
\end{array}\right\} B_{a}^{\jmath} N^{i}+\left\{\begin{array}{c}
h \\
j i
\end{array}\right\} \partial_{a} \partial_{t} x^{\jmath} N^{i}+\left\{\begin{array}{c}
h \\
j i
\end{array}\right\} B_{a}^{j} \partial_{t} N^{i} \\
& =\nabla_{a}\left(\partial_{t} N^{h}+\left\{\begin{array}{c}
h \\
j i
\end{array}\right\} \partial_{t} x^{\jmath} N^{i}\right)+K_{k j i} \partial_{t} x^{k} B_{a}^{j} N^{i}-\left\{\begin{array}{c}
h \\
j i
\end{array}\right\} \partial_{t} x^{j}\left(\partial_{a} N^{i}+\left\{\begin{array}{c}
i \\
l k
\end{array}\right\} B_{a}{ }^{l} N^{k}\right)
\end{aligned}
$$

where $K_{k j i}{ }^{h}$ is the curvature tensor of $M$. Thus we have

$$
\left(\partial_{t} \nabla_{a} N^{h}\right)_{0}=\nabla_{a} P^{h}+K_{k j i}{ }^{h} \xi^{k} B_{a}^{j} N^{i}-\left\{\begin{array}{l}
h \\
j i
\end{array}\right\} \xi^{j} \nabla_{a} N^{i}
$$

on $M^{\prime}$. From (3.4) and (3.5) we get

$$
D h_{b a}=-\nabla_{b} \xi^{\imath} g_{i n} \nabla_{a} N^{h}-B_{b}{ }^{h}\left(\nabla_{a} P_{h}+K_{k j i n} \xi^{k} B_{a}^{j} N^{i}\right) .
$$

From (3.6) we get

$$
\begin{aligned}
D\left(h_{e}^{e}\right) & =D\left(h_{b a} g^{b a}\right)=\left(D h_{b a}\right) g^{b a}-h^{b a} D_{b a} \\
& =-\nabla_{a} \xi^{i} \nabla^{a} N_{i}-B^{a}{ }_{i} \nabla_{a} P^{\imath}+K_{j i} \xi^{j} N^{i}-h_{b a} D^{b a},
\end{aligned}
$$

hence

$$
D h=\frac{1}{n}\left[-\nabla^{a} N_{i} \nabla_{a} \xi^{2}+K_{j i} N^{j} \xi^{2}+B^{a}{ }_{j} \nabla_{a}\left(B_{b}{ }^{j} N_{i} \nabla^{b} \xi^{i}\right)-2 h_{b}{ }^{a} B^{b}{ }_{i} \nabla_{a} \xi^{i}\right]
$$

where $h$ is the mean curvature.

Let us calculate deformation of the integral of the mean curvature over the hypersurface $M^{\prime}$, namely,

$$
\begin{aligned}
D H & =D \int_{M^{\prime}} h d V \\
& =\int_{M^{\prime}}\left(D h+\frac{1}{2} h g^{b a} D_{b a}\right) d V .
\end{aligned}
$$

Substituting (2.4) and (3.7) into the last member and using Green's theorem, we get

$$
\begin{gathered}
D H=\frac{1}{n} \int_{M^{\prime}}\left[-\nabla^{a} N_{i} \nabla_{a} \xi^{2}+K_{j i} N^{j} \xi^{2}+B^{a}{ }_{j} \nabla_{a}\left(B_{b}{ }^{j} N_{i} \nabla^{b} \xi^{i}\right)\right. \\
\left.-2 h_{b}{ }^{a} B^{b}{ }_{i} \nabla_{a} \xi^{2}+h_{b}{ }^{b} B^{a}{ }_{i} \nabla_{a} \xi^{i}\right] d V \\
=\frac{1}{n} \int_{M^{\prime}}\left[\left(\nabla_{a} \nabla^{a} N_{i}\right) \xi^{i}+K_{j i} N^{j} \xi^{i}-\left(\nabla_{a} B^{a}{ }_{j}\right) B_{b}{ }^{j} N_{i} \nabla^{b} \xi^{2}\right. \\
\left.+2 \nabla_{a}\left(h_{b}{ }^{a} B^{b}{ }_{i}\right) \xi^{i}-\nabla_{a}\left(h_{b}{ }^{b} B^{a}{ }_{i}\right) \xi^{i}\right] d V
\end{gathered}
$$


As we have

$$
\nabla_{a} \nabla^{a} N_{i}=-\left(\nabla_{a} h_{b}{ }^{a}\right) B^{b}{ }_{i}-h_{b}{ }^{a} h_{a}{ }^{b} N_{i},
$$

we get after some calculation

$$
\left.D H=\frac{1}{n} \int_{M^{\prime}}\left[\nabla_{b} h_{a}^{b}-\nabla_{a} h_{b}^{b}\right) B^{a}{ }_{i} \xi^{2}-\left\{\left(h_{a}^{a}\right)^{2}-h_{b}{ }^{a} h_{a}{ }^{b}\right\} N_{i} \xi^{2}+K_{j i} N^{j} \xi^{i}\right] d V .
$$

Substituting the equations

$$
\nabla_{b} h_{a}^{b}-\nabla_{a} h_{b}^{b}=-K_{j i} N^{j} B_{a}{ }^{2},
$$

which are derived from the equations of Codazzi, into the last member, we get

$$
D H=\frac{1}{n} \int_{M^{\prime}}\left[K_{k j} N^{k} N^{\jmath}-\left(h_{a}{ }^{a}\right)^{2}+h_{b}{ }^{a} h_{a}{ }^{b}\right] N_{i} \xi^{2} d V .
$$

If we use the scalar curvature ' $K$ of $M^{\prime}$, we can write (3.8) in the form

$$
D H=\frac{1}{n} \int_{M^{\prime}}\left(K-^{\prime} K-K_{k j} N^{k} N^{j}\right) N_{i} \xi^{\imath} d V
$$

for the equations of Gauss state that

$$
{ }^{\prime} K=K-2 K_{k h} N^{k} N^{h}+\left(h_{a}{ }^{a}\right)^{2}-h_{b}{ }^{a} h_{a}{ }^{b} .
$$

Thus we obtain the following proposition.

Proposition 3.2. A necessary and sufficient condition that the integral of the mean curvature over an orientable closed hypersurface $M^{\prime}$ in an orientable Riemannian manifold $M$ be critical for any infinitesimal deformation such that

$$
\int_{M^{\prime}} N_{i} \xi^{\imath} d V=0
$$

is that $K-{ }^{\prime} K-K_{k j} N^{k} N^{\jmath}$ be constant on $M^{\prime}$. A necessary and sufficient condition that the integral of the mean curvature over $M^{\prime}$ be critical for any infinitesimal deformation is that the following equation be satisfied on $M^{\prime}$,

$$
K-{ }^{\prime} K-K_{k j} N^{k} N^{\jmath}=0 .
$$

If $M$ is an Einstein space, $K$ and $K_{k j} N^{k} N^{\jmath}$ are constant. Hence we obtain the

Corollary. A necessary and sufficient condition that the integral of the mean curvature over an orientable closed hypersurface $M^{\prime}$ in an orientable Einstein space $M$ be critical for any infinitesimal deformation such that

$$
\int_{M^{\prime}} N_{i} \xi^{i} d V=0
$$


is that the scalar curvature ' $K$ of $M^{\prime}$ be constant.

\section{$\S 4$. Deformation of a closed hypersurface in Euclidean space.}

Let us consider the case where $M$ is a Euclidean space $E^{n+1}$.

Let $H(\lambda)$ and $H_{b}^{a}(\lambda)$ be defined by

$$
H(\lambda)=\operatorname{det}\left(h_{b}{ }^{a}-\lambda \delta_{b}^{a}\right),
$$

$$
H_{b}{ }^{a}(\lambda)\left(h_{c}{ }^{b}-\lambda \delta_{c}^{b}\right)=\delta_{c}^{a} H(\lambda)
$$

and put

$$
H(\lambda)=H_{n}+\lambda H_{n-1}+\cdots+\lambda^{n-1} H_{1}+(-\lambda)^{n} .
$$

From (4.2) we get

$$
\left(\nabla_{a} H_{b}{ }^{a}(\lambda)\right)\left(h_{c}{ }^{b}-\lambda \delta_{c}^{b}\right)+H_{b}{ }^{a}(\lambda) \nabla_{a} h_{c}{ }^{b}=\nabla_{c} H(\lambda) .
$$

As we have $\nabla_{a} h_{c}{ }^{b}=\nabla_{c} h_{a}^{b}$ by virtue of the Codazzi equations and as we have

$$
\nabla_{c} H(\lambda)=H_{b}{ }^{a}(\lambda) \nabla_{c} h_{a}{ }^{b},
$$

we get

$$
\left(\nabla_{a} H_{b}{ }^{a}(\lambda)\right)\left(h_{c}^{b}-\lambda \delta_{c}^{b}\right)=0,
$$

hence

$$
\nabla_{a} H_{b}{ }^{a}(\lambda)=0 .
$$

Differentiating $H_{b}{ }^{a}(\lambda)\left(h_{a}{ }^{c}-\lambda \delta_{a}^{c}\right)=\delta_{b}^{c} H(\lambda)$ covariantly, we also obtain

$$
\left(\nabla^{b} H_{b}^{a}(\lambda)\right)\left(h_{a}^{c}-\lambda \delta_{a}^{c}\right)+H_{b}{ }^{a}(\lambda) \nabla^{b} h_{a}^{c}=\nabla^{c} H(\lambda),
$$

hence

$$
\nabla^{b} H_{b}{ }^{a}(\lambda)=0
$$

by virtue of the Codazzi equations and $\nabla^{c} H(\lambda)=H_{b}{ }^{a}(\lambda) \nabla^{c} h_{a}{ }^{b}$.

Now let us calculate deformation of the integral of $H(\lambda)$ over $M^{\prime}$.

As we have (2.4) and

$$
\begin{aligned}
D H(\lambda) & =H_{a}^{b}(\lambda) D h_{b}{ }^{a} \\
& =H_{a}^{b}(\lambda)\left(g^{c a} D h_{b c}-h_{b}{ }^{d} g^{c a} D_{d c}\right),
\end{aligned}
$$

we get 


$$
\begin{aligned}
& D \int H(\lambda) d V \\
= & \int H(\lambda) \frac{1}{2} g^{d c} D_{d c} d V \\
& +\int H_{a}{ }^{b}(\lambda)\left(g^{c a} D h_{b c}-h_{b}{ }^{d} g^{c a} D_{d c}\right) d V \\
= & \int H(\lambda) B^{c}{ }_{i} \nabla_{c} \xi^{i} d V \\
& +\int H_{a}{ }^{b}(\lambda)\left(-\nabla^{a} \xi^{i} \nabla_{b} N_{i}-B^{a}{ }_{i} \nabla_{b} P^{\imath}-h_{b}{ }^{d} \nabla_{d} \xi^{i} B^{a}{ }_{i}-h_{b d} \nabla^{a} \xi^{i} B^{d}{ }_{i}\right) d V
\end{aligned}
$$

by virtue of (3.6) and $K_{k j i h}=0$. By Green's theorem the last member becomes

$$
\begin{aligned}
& =\int\left[-\left(\nabla_{c} H(\lambda)\right) B^{c}{ }_{i} \xi^{2}-h_{c}{ }^{c} H(\lambda) N_{i} \xi^{i}\right] d V \\
& +\int\left[H_{a}^{b}(\lambda)\left(\nabla^{a} \nabla_{b} N_{i}\right) \xi^{2}+H_{a}^{b}(\lambda) h_{b}{ }^{a} N_{i} P^{i}+\left(\nabla_{d} H_{a}{ }^{b}(\lambda)\right) h_{b}{ }^{d} B^{a}{ }_{i} \xi^{\imath}\right. \\
& \quad+H_{a}{ }^{b}(\lambda)\left(\nabla_{d} h_{b}{ }^{d}\right) B^{a}{ }_{i} \xi^{i}+H_{a}{ }^{b}(\lambda) h_{b}{ }^{d} h_{d}{ }^{a} N_{i} \xi^{i}+H_{a}{ }^{b}(\lambda)\left(\nabla^{a} h_{b d}\right) B^{d}{ }_{i} \xi^{2} \\
& \left.\quad+H_{a}{ }^{b}(\lambda) h_{b d} h^{a d} N_{i} \xi^{i}\right] d V
\end{aligned}
$$

where we have used (4.4) and (4.5).

As we have

$$
\begin{aligned}
\nabla^{a} \nabla_{b} N_{i} & =-\left(\nabla^{a} h_{b c}\right) B_{i}^{c}-h_{b c} h^{a c} N_{i}, \\
N_{i} P^{i} & =0,
\end{aligned}
$$

we get

$$
\begin{aligned}
& D \int H(\lambda) d V \\
= & \int\left[-\nabla_{c} H(\lambda)-H_{a}^{b}(\lambda) \nabla^{a} h_{b c}+\left(\nabla_{d} H_{c}^{b}(\lambda)\right) h_{b}{ }^{d}\right. \\
& \left.+H_{c}^{b}(\lambda) \nabla_{d} h_{b}^{d}+H_{a}^{b}(\lambda) V^{a} h_{b c}\right] B^{c}{ }^{c} \xi^{\imath} d V \\
& +\int\left[-h_{c}^{c} H(\lambda)-H_{a}^{b}(\lambda) h_{b c} h^{a c}+H_{a}^{b}(\lambda) h_{b}^{d} h_{d}^{a}+H_{a}^{b}(\lambda) h_{b d} h^{a d}\right] N_{i} \xi^{2} .
\end{aligned}
$$

We easily get 
158

$$
\begin{aligned}
& \left(\nabla_{d} H_{c}^{b}(\lambda)\right) h_{b}^{d} \\
= & \nabla_{d}\left(H_{c}^{b}(\lambda) h_{b}^{d}\right)-H_{c}^{b}(\lambda) \nabla_{d} h_{b}^{d} \\
= & \nabla_{d}\left\{H_{c}^{b}(\lambda)\left(h_{b}^{d}-\lambda \delta_{b}^{d}\right)+\lambda H_{c}^{d}(\lambda)\right\}-H_{c}^{b}(\lambda) \nabla_{d} h_{b}^{d} \\
= & \nabla_{d}\left(\delta_{c}^{d} H(\lambda)\right)-H_{c}^{b}(\lambda) \nabla_{d} h_{b}^{d} \\
= & \nabla_{c} H(\lambda)-H_{c}^{b}(\lambda) \nabla_{d} h_{b}{ }^{d},
\end{aligned}
$$

and the first integral containing $B^{c}{ }_{i} \xi^{i}$ vanishes.

Thus we obtain

$$
D \int H(\lambda) d V=\int\left[-h_{c}^{c} H(\lambda)+H_{a}^{b}(\lambda) h_{b c} h^{a c}\right] N_{i} \xi^{\imath} d V .
$$

On the other hand we have

$$
\begin{aligned}
& -h_{c}{ }^{c} H(\lambda)+H_{a}^{b}(\lambda) h_{b}{ }^{c} h_{c}{ }^{a} \\
= & -h_{c}{ }^{c} H(\lambda)+H_{a}{ }^{b}(\lambda)\left[\left(h_{b}{ }^{c}-\lambda \delta_{b}^{c}\right)\left(h_{c}{ }^{a}-\lambda \delta_{c}^{a}\right)+2 \lambda\left(h_{b}{ }^{a}-\lambda \delta_{b}^{a}\right)+\lambda^{2} \delta_{b}^{a}\right] \\
= & -h_{c}{ }^{c} H(\lambda)+\left(h_{c}{ }^{c}-n \lambda\right) H(\lambda)+2 n \lambda H(\lambda)+\lambda^{2} H_{a}{ }^{a}(\lambda) \\
= & n \lambda H(\lambda)+\lambda^{2} H_{a}{ }^{a}(\lambda)
\end{aligned}
$$

and

$$
\begin{aligned}
\frac{d}{d \lambda} H(\lambda) & =H_{b}{ }^{a}(\lambda) \frac{d}{d \lambda}\left(h_{a}^{b}-\lambda \delta_{a}^{b}\right) \\
& =-H_{a}^{a}(\lambda),
\end{aligned}
$$

hence

$$
\begin{aligned}
& -h_{c}^{c} H(\lambda)+H_{a}^{b}(\lambda) h_{b c} h^{a c} \\
= & n \lambda H(\lambda)-\lambda^{2} \frac{d}{d \lambda} H(\lambda) \\
= & \sum_{m=0}^{n}(n-m) H_{n-m} \lambda^{m+1}
\end{aligned}
$$

where we have used (4.3).

Substituting this identity into (4.6) we get

$$
\begin{aligned}
& \sum_{m=0}^{n} D \int H_{n-m} d V \lambda^{m} \\
= & \sum_{m=0}^{n}(n-m) \int H_{n-m} N_{i} \xi^{\imath} d V \lambda^{m+1},
\end{aligned}
$$


hence

$$
D \int H_{n} d V=0
$$

$$
D \int H_{n-m} d V=(n-m+1) \int H_{n-m+1} N_{i} \xi^{\imath} d V \quad(m=1, \cdots, n) .
$$

Thus we obtain the following theorem.

THEOREM 4. 1. Let $M^{\prime}$ be a closed hypersurface in a Euclidean space $E^{n+1}$ and let $H(\lambda)$ and $H_{m}$ be defined by (4.1) and (4.3). If $\xi^{h}$ is a vector field of an infinitesimal deformation of the hypersurface $M^{\prime}$ and $D$ is the symbol of the deformation induced by $\xi^{h}$, then $H_{0}, H_{1}, \cdots, H_{n}$ satisfy (4.7) and (4. 8).

(4. 7) states that the integral of $\operatorname{det}\left(h_{b}{ }^{a}\right)$ over the hypersurface $M^{\prime}$ is a topological invariant. This is a well-known fact, the integral being equal to the volume integral of $S^{n}$ multiplied by the degree of mapping of the Gauss map $\varphi: M^{\prime} \rightarrow S^{n}$ induced by the unit normal vector field $N^{h}$.

Department of Applied Mathematics, Faculty of Engineering, YOKOHAMA NATIONAL UNIVERSITY. 